\title{
Colors and magnitudes of quasar host galaxies at high redshift
}

\author{
Susan E. Ridgway ${ }^{1}$, Tim Heckman ${ }^{1}$ \\ and Mark Lacy ${ }^{2}$ \\ ${ }^{1}$ Dept. of Physics and Astronomy, Johns Hopkins University, 3400 N. Charles St., Baltimore \\ MD, USA email: ridgway@pha.jhu.edu, heckman@pha.jhu.edu \\ ${ }^{2}$ Spitzer Science Center, MS220-6, Caltech, 1200 E. California Blvd, Pasadena, CA 91125, \\ USA email: mlacy@ipac.caltech.edu
}

\begin{abstract}
We discuss the results so far of a program to image in the rest-frame optical and near-UV the host galaxies of a sample of 5 high redshift $(z \sim 2-3)$ radio-quiet quasars with nuclear luminosities lower than most samples studied at high $z$. We found that in the rest-frame optical the hosts have luminosities of about $L_{*}$, comparable to the Lyman break galaxies at similar redshifts. From the rest-frame UV imaging, we can derive optical-UV colors, and find they show a great deal of variation, but may be somewhat redder and less starforming than the LBGs. We are supplementing this work at high $z$ with study of the hosts of $z \sim 1$ radio-quiet quasars with similar low nuclear luminosities.
\end{abstract}

\section{Introduction}

Understanding the links between the formation and the evolution of the AGN population and the formation and evolution of galaxies requires investigation of the relationship between these populations at high redshift, where the quasar luminosity function is reaching a maximum. We have been studying the properties of the hosts of lower-luminosity radio-quiet quasars (RQQs) at high redshift $(z \sim 2.5)$ at rest-frame optical and have found the hosts to be only about $L_{*}$. We discuss here, in addition, the results of subsequent WFPC2 imaging of these same sources at rest-frame $\lambda \sim 2000 \AA$. The resulting optical-UV colors allow us to make a rough judgement of the starforming properties of the galaxies.

In contrast, at $z \sim 1$, most high-luminosity quasars seem to have very massive hosts, more comparable to those of the radio-loud objects. To determine whether this difference is due to evolution or to the difference in AGN luminosity, we have recently started a deep ACS imaging program of a sample of lower-luminosity radio-quiet quasar hosts at $z \sim 1$. This will allow us to study the host galaxy mass as a function of AGN luminosity (and therefore black hole mass) and as a function of redshift.

\section{The High $z$ Sample}

We have selected 5 quasars with $\mathrm{B}>21.5$ at $z \sim 2-3$ with absolute nuclear magnitudes comparable to most low- $z$ quasar samples. We made infrared observations of these sources with HST's NICMOS camera, in the F165M and F160W filters, at a scale of 0 ". 075 pixel $^{-1}$. The optical observations were made with the PC aperture of the WFPC2 camera, providing a scale of 0.046 pixel $^{-1}$, in the F606W filter. We observed stars interleaved with the quasars to enable characterization of the point-spread-function (PSF) and have made a direct two-dimensional PSF subtraction through an iterative fitting method. 


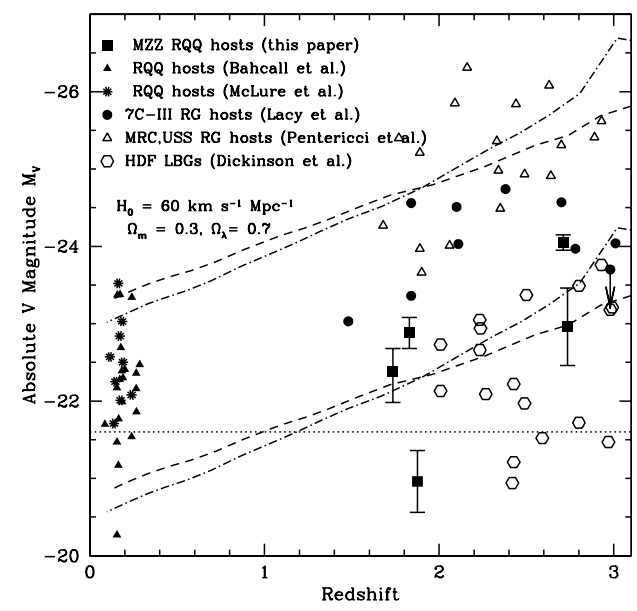

Figure 1. The total absolute rest-frame $V$ magnitudes versus redshift for our quasar hosts (squares) and other samples of host galaxies.

\section{Results}

The rest-frame optical imaging revealed host galaxies with rest-frame absolute magnitudes of about 0.2 to $4 \mathrm{~L}_{*}$. (The details of the analysis are discussed in Ridgway et al. 2001). In Figure 1, we show the absolute rest-frame V magnitudes for these RQQ hosts vs. those of various other samples of high $z$ galaxies. We also determine half-light radii for the hosts and find they are compact, $<4 \mathrm{kpc}$ at these redshifts.

Detection of extended emission in the rest-frame UV is more difficult; however, we were able to detect extended emission around the nucleus in 4 of the 5 cases. We determine the global colors of the galaxies with simple aperture photometry, and compare our derived colors to the LBGs (Dickinson 2000). We find that although the color distributon of our sample of objects overlaps that of the LBGs, the average color of the host galaxies is redder than those of the LBGs.

\section{Discussion}

We present here the results of a rest-frame optical and UV imaging survey of a sample of 5 RQQ at $z \sim 2-3$, the peak of the quasar luminosity function. It now seems fairly conclusive from this work and many others that RQQs at high $z$ are associated with $L_{*}$ galaxies, in contrast to the bright, many times $L_{*}$ galaxies associated with radio-loud objects at these redshifts (Ridgway et al. 2001, Kukula et al. 2001, Rix et al. 2001). These results are roughly consistent with the hierarchical assembly theories of galaxy formation (Kauffmann \& Haehnelt 2000). However, our rest-frame UV imaging suggests that these hosts are not as actively star-forming as the Lyman break galaxies at similar redshifts.

\section{References}

Dickinson, M. 2000, Philosophical Transactions of the Royal Society of London, 358, 2001

Kauffmann, G., \& Haehnelt, M. 2000, MNRAS, 311, 576

Kukula, M. et al. 2001, MNRAS, 326, 1533

Ridgway, S. E., Heckman, T. M., Calzetti, D., \& Lehnert, M. 2001, ApJ, 550, 122

Rix, H.-W. et al. 2001, in Gravitational Lensing: Recent Progress and Future Goals, (eds. T. G. Brainerd and C. S. Kochanek), ASP Conf. Ser., 237, 169 